PROCEEDINGS OF THE

AMERICAN MATHEMATICAL SOCIETY

Volume 138, Number 9, September 2010, Pages 3037-3046

S 0002-9939(10)10381-5

Article electronically published on April 21, 2010

\title{
HOPF FORMULAS FOR EQUIVARIANT INTEGRAL HOMOLOGY OF GROUPS
}

\author{
HVEDRI INASSARIDZE AND EMZAR KHMALADZE
}

(Communicated by Alexander N. Dranishnikov)

\begin{abstract}
By using purely algebraic methods of $n$-fold Čech derived functors, the higher equivariant integral group homology is investigated from the Hopf formulas point of view.
\end{abstract}

The study of group actions on algebraic objects has many important applications in homotopy theory and $K$-theory (see for instance [3, 10, 13, 15]). In [11 the first author introduced and studied a natural equivariant version of the classical Eilenberg-Mac Lane (co)homology theory of groups, which can be considered as a part of relative homological algebra. The cohomological characterization of equivariant extensions with operators that are splitting with respect to group actions has motivated the development of this theory, which differs from the (co)homology theory of groups with operators developed in [5, 6. motivated by the classification problem of graded categorical groups. Analogues of much of the theory of the classical homological algebra of groups have been obtained in that equivariant setting such as (co)chain and cotriple presentations, the Hopf formula, exact (co)homology sequences, and the Tate cohomology of groups. Moreover, the relationship with the equivariant cohomology of spaces was established, and it was shown that the well known isomorphism of Milnor's $K$-group $K_{2}(A)$ with the second integral homology $H_{2}(E(A))$ of the elementary group $E(\mathrm{~A})$ of a unital ring $A$ remains true for arbitrary rings by taking into account the action on $E(A)$ of the Steinberg group $S t(\mathbb{Z})$ of the ring $\mathbb{Z}$ of integers (see [11, Corollary 24]).

As promised we continue the investigation of equivariant homology theory of H. Inassaridze 11]. Namely, using the purely algebraic method of $n$-fold Čech derived functors developed in 7, 12, we provide higher Hopf type formulas for the equivariant integral group homology (Theorem 12), extending the Hopf formula for the second equivariant homology [11] and recovering the Brown-Ellis higher Hopf formulas [2], when the group actions are trivial.

We set up the following notation. Throughout $\Gamma$ is a fixed group. $\mathcal{G}_{\Gamma}$ denotes the category of $\Gamma$-groups whose objects are groups enriched with a (left) $\Gamma$-action and morphisms are $\Gamma$-equivariant group homomorphisms, or $\Gamma$-homomorphisms for

Received by the editors July 10, 2009.

2010 Mathematics Subject Classification. Primary 18G10, 18G50.

Key words and phrases. Hopf formula, equivariant homology, cotriple homology, projective simplicial resolution, crossed $n$-cube, Čech derived functor.

The authors were supported by the Volkswagen Foundation, Ref.: I/84 328, INTAS, Ref.: 06-1000017-8609; and the Georgian National Science Foundation, Ref.: ST06/3-004.

(C)2010 American Mathematical Society 
short. The categories of groups, abelian groups and $\Gamma$-sets are denoted by $\mathcal{G}, \mathcal{A} b$ and $\mathcal{S}_{\Gamma}$, respectively. For a non-negative integer $n$ we denote by $\langle n\rangle$ the set of the first $n$ natural numbers $\{1, \cdots, n\}$. For any set $A$ its cardinality will be denoted by $|A|$.

We begin by formulating in the $\Gamma$-equivariant framework some well known notions and recall some facts from 11 needed for our future purpose.

For any $\Gamma$-group $H$, the group $A u t(H)$ of automorphisms of the group $H$ is a $\Gamma$-group with the diagonal $\Gamma$-action given by

$$
\left({ }^{\sigma} f\right)(h)={ }^{\sigma} f\left({ }^{\sigma^{-1}} h\right)
$$

for all $\sigma \in \Gamma, f \in A u t(H)$ and $h \in H$.

Definition 1. A $\Gamma$-equivariant action of a $\Gamma$-group $G$ on a $\Gamma$-group $H$ is a $\Gamma$ homomorphism $G \rightarrow \operatorname{Aut}(H)$, or alternatively, this is a group action of $G$ on $H$ such that

$$
{ }^{\sigma}\left({ }^{g} h\right)=\left({ }^{\sigma} g\right)\left({ }^{\sigma} h\right)
$$

for all $\sigma \in \Gamma, g \in G$ and $h \in H$.

Note that if $H$ is an abelian group in the previous definition, then it inherits a structure of a $\Gamma$-equivariant $G$-module [6, 5, 11.

Let $G$ be a $\Gamma$-group acting on a $\Gamma$-group $H$. Then the semi-direct product of groups $H \rtimes G$ is a $\Gamma$-group with the $\Gamma$-action given by

$$
{ }^{\sigma}(h, g)=\left({ }^{\sigma} h,{ }^{\sigma} g\right)
$$

for $\sigma \in \Gamma, h \in H$ and $g \in G$.

Given a $\Gamma$-group $G$ and a normal $\Gamma$-subgroup $H$ of $G$, we denote by $[G]_{\Gamma}$ the subgroup of $G$ generated by the elements of the form ${ }^{\sigma} g g^{-1}$, and by $[G, H]_{\Gamma}$ the subgroup of $H$ generated by the elements $g^{\sigma} h g^{-1} h^{-1}$, where $\sigma \in \Gamma, g \in G, h \in H$. It is easy to check that $[G]_{\Gamma}$ is a normal $\Gamma$-subgroup of $G$ as well as that $[G, H]_{\Gamma}$ is a normal $\Gamma$-subgroup of $H$ and $[G, H]_{\Gamma}=[G, H][H]_{\Gamma}$. Moreover, the quotient $H /[G, H]_{\Gamma}$ is an abelian group on which $\Gamma$ acts trivially. For $H=G$ this quotient defines the $\Gamma$-abelianization functor $\mathfrak{A b}_{\Gamma}: \mathcal{G}_{\Gamma} \rightarrow \mathcal{A} b, \mathfrak{A b}_{\Gamma}(G)=G /[G, G]_{\Gamma}$, which is left adjoint to the inclusion $\mathcal{A} b \hookrightarrow \mathcal{G}_{\Gamma}$.

In 11 the (co)cycle description of the equivariant (co)homology of groups is given and its cotriple (co)homology interpretation as well. In particular the equivariant integral homology groups $H_{n}^{\Gamma}(G), n \geq 0$, of a $\Gamma$-group $G$ are described via cotriple derived functors of the $\Gamma$-abelianization functor $\mathfrak{A b}_{\Gamma}$. More precisely, the usual forgetful functor $\mathcal{U}: \mathcal{G}_{\Gamma} \rightarrow \mathcal{S}_{\Gamma}$ has a left adjoint $\mathcal{F}$, which assigns to any $\Gamma$-set $X$ the free group $\mathcal{F}(X)$ on the set $X$ with the $\Gamma$-action given by ${ }^{\sigma}|x|=\left|{ }^{\sigma} x\right|, x \in X$, $\sigma \in \Gamma$. The adjoint pair of functors $\mathcal{S}_{\Gamma} \underset{\mathcal{U}}{\stackrel{\mathcal{F}}{\gtrless}} \mathcal{G}_{\Gamma}$ induces a cotriple $\mathbb{F}=(\mathbb{F}, \tau, \delta)$ in $\mathcal{G}_{\Gamma}$ in the obvious way: $\mathbb{F}=\mathcal{F} \mathcal{U}, \tau: \mathbb{F} \rightarrow i d_{\mathcal{G}_{\Gamma}}$ is the counit and $\delta=\mathcal{F} u \mathcal{U}$, where $u: i d_{\mathcal{S}_{\Gamma}} \rightarrow \mathcal{U F}$ is the unit of the adjunction. The resulting $\mathbb{F}$-cotriple resolution of $G$ is the simplicial $\Gamma$-group $\mathbb{F}_{*}(G)$ with $\mathbb{F}_{0}(G)=\mathbb{F}(G), \mathbb{F}_{n}(G)=\mathbb{F}^{n+1}(G)=\mathbb{F}\left(\mathbb{F}^{n}(G)\right.$ ), $d_{i}^{n}=\mathbb{F}^{i}\left(\tau_{\mathbb{F}^{n-i}}\right), s_{i}^{n}=\mathbb{F}^{i}\left(\delta_{\mathbb{F}^{n-i}}\right), 0 \leq i \leq n$. Applying the functor $\mathfrak{A b}_{\Gamma}$ dimensionwise to $\mathbb{F}_{*}(G)$ we obtain the simplicial abelian group $\mathfrak{A b}_{\Gamma} \mathbb{F}_{*}(G)$. According to [11, Theorem 10] we have

$$
H_{n}^{\Gamma}(G)=\pi_{n-1}\left(\mathfrak{A b}_{\Gamma} \mathbb{F}_{*}(G)\right), \quad n \geq 1 .
$$


A $\Gamma$-group $F$ is called $(\mathbb{F}$-) projective if $F$ is a retract of some value of $\mathbb{F}$, or equivalently, if there is a $\Gamma$-homomorphism $s: F \rightarrow \mathbb{F}(F)$ such that $\tau_{F} s=i d_{F}$. If that is the case, it is well known that the simplicial object $\mathbb{F}_{*}(F)$ admits a contraction and thus $H_{n}^{\Gamma}(F)=0$, for $n \geq 2$.

Remark 2. For computing the equivariant integral homology of a $\Gamma$-group $G$, we will use 'non-standard' resolutions in the sense of Barr-Beck 1], in particular, the so-called projective simplicial resolution of $G$, defined as an augmented simplicial $\Gamma$-group $F_{*} \rightarrow G$ such that $F_{n}$ is projective for all $n \geq 0$, and the induced simplicial $\operatorname{map} \mathcal{U}\left(F_{*}\right) \rightarrow \mathcal{U}(G)$ is a homotopy equivalence (of simplicial $\Gamma$-sets). The $\mathbb{F}$-cotriple simplicial resolution of $G$ is a projective simplicial resolution and by [1, 5.3], if $F_{*} \rightarrow G$ is any one of them, then there is an isomorphism $H_{n}^{\Gamma}(G)=\pi_{n-1}\left(\mathfrak{A} \mathfrak{b}_{\Gamma} F_{*}\right)$, $n \geq 1$.

To realize the algebraic proof of higher Hopf formulas and their further generalizations, one of the main tools used in [7] is the theory of crossed $n$-cubes of groups introduced by Ellis and Steiner [9]. An equivariant analog of the notion of crossed $n$-cubes takes the following form.

Definition 3. A crossed $n$-cube of $\Gamma$-groups is a family $\mathcal{M}=\left\{\mathcal{M}_{A}: A \subseteq\langle n\rangle\right\}$ of $\Gamma$-groups together with $\Gamma$-homomorphisms $\mu_{i}: \mathcal{M}_{A} \rightarrow \mathcal{M}_{A \backslash\{i\}}$ for $i \in\langle n\rangle$, $A \subseteq\langle n\rangle$ and functions $\kappa: \mathcal{M}_{A} \times \mathcal{M}_{B} \longrightarrow \mathcal{M}_{A \cup B}$ for $A, B \subseteq\langle n\rangle$, such that if ${ }^{a} b$ denotes $\kappa(a, b) \cdot b$ for $a \in \mathcal{M}_{A}$ and $b \in \mathcal{M}_{B}$ with $A \subseteq B$, then for all $a, a^{\prime} \in \mathcal{M}_{A}$, $b, b^{\prime} \in \mathcal{M}_{B}, c \in \mathcal{M}_{C}, \sigma \in \Gamma$ and $i, j \in\langle n\rangle$, the following conditions hold:

$$
\begin{aligned}
& \mu_{i}(a)=a \text { if } i \notin A, \\
& \mu_{i} \mu_{j}(a)=\mu_{j} \mu_{i}(a), \\
& \mu_{i} \kappa(a, b)=\kappa\left(\mu_{i}(a), \mu_{i}(b)\right), \\
& \kappa(a, b)=\kappa\left(\mu_{i}(a), b\right)=\kappa\left(a, \mu_{i}(b)\right) \text { if } i \in A \cap B, \\
& \kappa\left(a, a^{\prime}\right)=\left[a, a^{\prime}\right], \\
& \kappa(a, b)=\kappa(b, a)^{-1}, \\
& \kappa(a, b)=1 \text { if } a=1 \text { or } b=1, \\
& \kappa\left(a a^{\prime}, b\right)={ }^{a} \kappa\left(a^{\prime}, b\right) \kappa(a, b), \\
& \kappa\left(a, b b^{\prime}\right)=\kappa(a, b){ }^{b} \kappa\left(a, b^{\prime}\right), \\
& { }^{a} \kappa\left(\kappa\left(a^{-1}, b\right), c\right)^{c} \kappa\left(\kappa\left(c^{-1}, a\right), b\right){ }^{b} \kappa\left(\kappa\left(b^{-1}, c\right), a\right)=1, \\
& { }^{a} \kappa(b, c)=\kappa\left({ }^{a} b{ }^{a}{ }^{a} c\right) \text { if } A \subseteq B \cap C, \\
& { }^{\sigma} \kappa(a, b)=\kappa\left({ }^{\sigma} a,{ }^{\sigma} b\right) .
\end{aligned}
$$

A morphism of crossed $n$-cubes of $\Gamma$-groups, $\alpha: \mathcal{M} \rightarrow \mathcal{N}$, is a family of $\Gamma$ homomorphisms $\left\{\alpha_{A}: \mathcal{M}_{A} \rightarrow \mathcal{N}_{A}, A \subseteq\langle n\rangle\right\}$ commuting with the functions $\mu_{i}$ and $\kappa$. The resulting category of crossed $n$-cubes of $\Gamma$-groups will be denoted by $\mathcal{X}^{n} \mathcal{G}_{\Gamma}$.

Example 4. Let $F$ be a $\Gamma$-group and $R_{1}, \ldots, R_{n}$ be normal $\Gamma$-subgroups of $F$. Let $\mathcal{M}_{A}=\bigcap_{i \in A} R_{i}$ for $A \subseteq\langle n\rangle$ (here $\mathcal{M}_{\emptyset}$ is understood to mean $F$ ); if $i \in\langle n\rangle$, define $\mu_{i}: \mathcal{M}_{A} \rightarrow \mathcal{M}_{A \backslash\{i\}}$ to be the inclusion and given $A, B \subseteq\langle n\rangle$, let $\kappa: \mathcal{M}_{A} \times \mathcal{M}_{B} \rightarrow \mathcal{M}_{A \cup B}$ be given by the commutator: $\kappa(a, b)=[a, b]$ for $a \in \mathcal{M}_{A}$, $b \in \mathcal{M}_{B}$. Then $\left\{\mathcal{M}_{A}: A \subseteq\langle n\rangle, \mu_{i}, \kappa\right\}$ is a crossed $n$-cube, called the inclusion crossed $n$-cube given by the normal $(n+1)$-ad of $\Gamma$-groups $\left(F ; R_{1}, \ldots, R_{n}\right)$. 
Recall from [7] (see also [4) that the crossed $n$-cube of groups is abelian if the $\kappa$ maps are trivial. Let us denote by $\mathcal{A} b \mathcal{X}^{n} \mathcal{G}$ the category of abelian crossed $n$-cubes of groups. There is a $\Gamma$-abelianization functor

$$
\mathfrak{A b}_{\Gamma}^{n}: \mathcal{X}^{n} \mathcal{G}_{\Gamma} \rightarrow \mathcal{A} b \mathcal{X}^{n} \mathcal{G}
$$

defined for any crossed $n$-cube of $\Gamma$-groups $\mathcal{M}=\left\{\mathcal{M}_{A}: A \subseteq\langle n\rangle, \mu_{i}, \kappa\right\}$ by

$$
\mathfrak{A b}_{\Gamma}^{n}(\mathcal{M})_{A}=\frac{\mathcal{M}_{A}}{\prod_{B \cup C=A} D_{\Gamma}(B, C)},
$$

where $D_{\Gamma}(B, C)$ is the subgroup of $\mathcal{M}_{A}$ generated by the elements of the form $\sigma_{a a^{-1}}$ and $\kappa(b, c)$, where $\kappa: \mathcal{M}_{B} \times \mathcal{M}_{C} \rightarrow \mathcal{M}_{B \cup C=A}, \sigma \in \Gamma, a \in \mathcal{M}_{A}, b \in \mathcal{M}_{B}$ and $c \in \mathcal{M}_{C}$. The homomorphism $\widetilde{\mu}_{i}: \mathfrak{A b}_{\Gamma}^{n}(\mathcal{M})_{A} \rightarrow \mathfrak{A b}_{\Gamma}^{n}(\mathcal{M})_{A \backslash\{i\}}$, for $i \in\langle n\rangle$ and the function $\widetilde{\kappa}: \mathfrak{A b}_{\Gamma}^{n}(\mathcal{M})_{A} \times \mathfrak{A b}_{\Gamma}^{n}(\mathcal{M})_{B} \rightarrow \mathfrak{A b}_{\Gamma}^{n}(\mathcal{M})_{A \cup B}$, for $A, B \subseteq\langle n\rangle$, are induced by the homomorphism $\mu_{i}$ and the map $\kappa$, respectively.

Note that for the trivial group $\Gamma=1$ the functor $\mathfrak{A b}_{\Gamma}^{n}$ is the usual abelianization functor from the category $\mathcal{X}^{n} \mathcal{G}$ of crossed $n$-cubes of groups to the category $\mathcal{A} b \mathcal{X}^{n} \mathcal{G}$ of abelian group objects in $\mathcal{X}^{n} \mathcal{G}$ (see 4]).

For $n=1$, Definition 3 can be simplified considerably, and we arrive at the following equivalent notion: A crossed module of $\Gamma$-groups $(H, G, \mu)$ is a $\Gamma$-homomorphism $\mu: H \rightarrow G$ together with the $\Gamma$-equivariant action of $G$ on $H$ such that

(i) $\mu\left({ }^{g} h\right)=g \mu(h) g^{-1}$,

(ii) ${ }^{\mu(h)} h^{\prime}=h h^{\prime} h^{-1}$

for all $h, h^{\prime} \in H, g \in G$. In particular, $(H, G, \mu)$ is a crossed module of groups, and we can form its nerve [14], which is the simplicial group $E_{\Gamma}^{1}(H, G, \mu)_{*}$, where $E_{\Gamma}^{1}(H, G, \mu)_{k}=H \rtimes(\cdots(H \rtimes G) \cdots)$ with $k$ semi-direct factors of $H$, and the face and degeneracy homomorphisms are given by

$$
\begin{aligned}
& d_{0}\left(h_{1}, \cdots, h_{k}, g\right)=\left(h_{2}, \cdots, h_{k}, g\right), \\
& d_{i}\left(h_{1}, \cdots, h_{k}, g\right)=\left(h_{1}, \cdots, h_{i} h_{i+1}, \cdots, h_{k}, g\right), \quad 0 \leq i \leq k, \\
& d_{k}\left(h_{1}, \cdots, h_{k}, g\right)=\left(h_{1}, \cdots, h_{k-1}, \mu\left(h_{k}\right) g\right), \\
& s_{i}\left(h_{1}, \cdots, h_{k}, g\right)=\left(h_{1}, \cdots, h_{i}, 1, h_{i+1}, \cdots, h_{k}, g\right), \quad 0 \leq i \leq k .
\end{aligned}
$$

It is clear that $E_{\Gamma}^{1}(H, G, \mu)_{*}$ is a simplicial $\Gamma$-group with the above described $\Gamma$ action on the semi-direct products and it will be called the nerve of the crossed module of $\Gamma$-groups.

Given a crossed $n$-cube of $\Gamma$-groups $\mathcal{M}$, applying the nerve of crossed modules of $\Gamma$-groups $E_{\Gamma}^{1}$ in the $n$-independent directions, this construction leads naturally to an $n$-simplicial $\Gamma$-group, called the multinerve of $\mathcal{M}$. Taking the diagonal of this $n$-simplicial $\Gamma$-group gives a simplicial $\Gamma$-group denoted by $E_{\Gamma}^{n}(\mathcal{M})_{*}$.

Now we show that the $\Gamma$-abelianization of an inclusion crossed $n$-cube of $\Gamma$-groups commutes with the functor $E_{\Gamma}^{n}$.

Proposition 5. Let $\mathcal{M}$ be an inclusion crossed $n$-cube given by a normal $(n+1)$-ad of $\Gamma$-groups $\left(F ; R_{1}, \cdots, R_{n}\right)$. Then there is an isomorphism of simplicial groups

$$
\mathfrak{A} \mathfrak{b}_{\Gamma} E_{\Gamma}^{n}(\mathcal{M})_{*} \cong E_{\Gamma}^{n}\left(\mathfrak{A b}_{\Gamma}^{n}(M)\right)_{*} .
$$


Proof. There is a natural morphism of crossed $n$-cubes of $\Gamma$-groups $\mathcal{M} \rightarrow \mathfrak{A b}_{\Gamma}^{n}(\mathcal{M})$ inducing the natural surjection of simplicial $\Gamma$-groups $E_{\Gamma}^{n}(\mathcal{M})_{*} \stackrel{\Delta_{*}^{n}}{\longrightarrow} E_{\Gamma}^{n}\left(\mathfrak{A b}_{\Gamma}^{n}(\mathcal{M})\right)_{*}$ given by

$$
\Delta_{k}^{n}\left(x_{1}, \cdots, x_{l}\right)=\left(\overline{x_{1}}, \cdots, \overline{x_{l}}\right),
$$

where the bar denotes a coset, $\left(x_{1}, \cdots, x_{l}\right) \in E_{\Gamma}^{n}(\mathcal{M})_{k}=\bigcap_{i \in A_{1}} R_{i} \rtimes \cdots$ $\rtimes \bigcap_{i \in A_{l}} R_{i}, k \geq 0, l=(k+1)^{n}$ and $A_{1}, \cdots, A_{l} \subseteq\langle n\rangle$. We only need to show that

$$
\operatorname{Ker} \Delta_{k}^{n}=\left[E_{\Gamma}^{n}(\mathcal{M})_{k}, E_{\Gamma}^{n}(\mathcal{M})_{k}\right]_{\Gamma}
$$

One easily notices that

$$
\operatorname{Ker} \Delta_{k}^{n}=\prod_{B_{1} \cup C_{1}=A_{1}} D_{\Gamma}\left(B_{1}, C_{1}\right) \rtimes \cdots \rtimes \prod_{B_{l} \cup C_{l}=A_{l}} D_{\Gamma}\left(B_{l}, C_{l}\right) .
$$

Then, to check the inclusion $\operatorname{Ker} \Delta_{k}^{n} \subseteq\left[E_{\Gamma}^{n}(\mathcal{M})_{k}, E_{\Gamma}^{n}(\mathcal{M})_{k}\right]_{\Gamma}$, it is sufficient to show that

$$
1 \rtimes \cdots \rtimes 1 \rtimes \prod_{B_{s} \cup C_{s}=A_{s}} D_{\Gamma}\left(B_{s}, C_{s}\right) \rtimes 1 \cdots \rtimes 1 \subseteq\left[E_{\Gamma}^{n}(\mathcal{M})_{k}, E_{\Gamma}^{n}(\mathcal{M})_{k}\right]_{\Gamma}
$$

for all $s \in\left\langle(k+1)^{n}\right\rangle$. Since any generator of $D_{\Gamma}\left(B_{s}, C_{s}\right)$ has the form ${ }^{\sigma} a a^{-1}$ or $[b, c]$, where $\sigma \in \Gamma, a \in \mathcal{M}_{A_{s}}, b \in \mathcal{M}_{B_{s}}$ and $c \in \mathcal{M}_{C_{s}}$, the inclusion follows from [7, Proposition 11] and the equality $\left(1, \cdots, 1,{ }^{\sigma} a a^{-1}, 1, \cdots, 1\right)={ }^{\sigma}(1, \cdots, 1, a, 1, \cdots, 1)$ $(1, \cdots, 1, a, 1, \cdots, 1)^{-1}$.

To show the inverse inclusion $\left[E_{\Gamma}^{n}(\mathcal{M})_{k}, E_{\Gamma}^{n}(\mathcal{M})_{k}\right]_{\Gamma} \subseteq \operatorname{Ker} \Delta_{k}^{n}$, we notice that any generator of $\left[E_{\Gamma}^{n}(\mathcal{M})_{k}, E_{\Gamma}^{n}(\mathcal{M})_{k}\right]_{\Gamma}$ has the form ${ }^{\sigma} \omega \omega^{-1}$ or $\left[\omega_{1}, \omega_{2}\right]$, where $\sigma \in \Gamma$, $\omega, \omega_{1}, \omega_{2} \in E_{\Gamma}^{n}(\mathcal{M})_{k}$. Again by using [7, Proposition 11] we have $\left[\omega_{1}, \omega_{2}\right] \in \operatorname{Ker} \Delta_{k}^{n}$. Now, an element $\omega \in E_{\Gamma}^{n}(\mathcal{M})_{k}$ can be written as $\omega=\prod_{s=1}^{(k+1)^{n}}\left(1, \cdots, 1, x_{s}, 1, \cdots, 1\right)$, where $x_{s} \in \bigcap_{i \in A_{s}} R_{i}$. Then

$$
\begin{aligned}
{ }^{\sigma} \omega \omega^{-1}=\prod_{s=1}^{(k+1)^{n}}\left(1, \cdots,{ }^{\sigma} x_{s} x_{s}^{-1}, \cdots, 1\right) \\
\\
\quad \prod_{s=1}^{(k+1)^{n}}\left(1 \rtimes \cdots \rtimes 1 \rtimes \prod_{B_{s} \cup C_{s}=A_{s}} D_{\Gamma}\left(B_{s}, C_{s}\right) \rtimes 1 \cdots \rtimes 1\right) \subseteq \operatorname{Ker} \Delta_{k}^{n}
\end{aligned}
$$

and the assertion follows.

The diagonal of the multinerve of crossed $n$-cubes of $\Gamma$-groups is closely related to the $n$-fold Cech derivatives of functors from the category of $\Gamma$-groups to the category of groups, which we consider immediately below, whilst the general situation has been dealt with in [12.

Let us consider the set $\langle n\rangle$. The subsets of $\langle n\rangle$ are ordered by inclusion. This ordered set determines in the usual way a category $\mathcal{C}_{n}$. For every pair $(A, B)$ of subsets with $A \subseteq B \subseteq\langle n\rangle$, there is the unique morphism $\rho_{B}^{A}: A \rightarrow B$ in $\mathcal{C}_{n}$. Any morphism in $\mathcal{C}_{n}$, not an identity, is generated by $\rho_{A \cup\{j\}}^{A}$ for all $A \subseteq\langle n\rangle, A \neq\langle n\rangle$ and $j \in\langle n\rangle \backslash A$.

An $n$-cube of $\Gamma$-groups is a functor $\mathfrak{F}: \mathcal{C}_{n} \rightarrow \mathcal{G}_{\Gamma}$. A morphism between n-cubes $\mathfrak{F}, \mathfrak{F}^{\prime}: \mathcal{C}_{n} \rightarrow \mathcal{G}_{\Gamma}$ is a natural transformation $\kappa: \mathfrak{F} \rightarrow \mathfrak{F}^{\prime}$. 
Example 6. Let $\left(G_{*}, d_{0}^{0}, G\right)$ be an augmented simplicial object in the category $\mathcal{G}_{\Gamma}$. A natural $n$-cube of $\Gamma$-groups $\mathfrak{F}^{(n)}=\mathfrak{F}^{(n)}\left(G_{*}, d_{0}^{0}, G\right): \mathcal{C}_{n} \rightarrow \mathcal{G}_{\Gamma}, n \geq 1$ is defined in the following way:

$$
\begin{gathered}
\mathfrak{F}^{(n)}(A)=G_{n-1-|A|} \text { for all } A \subseteq\langle n\rangle, \\
\mathfrak{F}^{(n)}\left(\rho_{A \cup\{j\}}^{A}\right)=d_{k-1}^{n-1-|A|} \text { for all } A \neq\langle n\rangle, j \in\langle n\rangle \backslash A,
\end{gathered}
$$

where $G_{-1}=G$ and $k \in\langle n-|A|\rangle$ is the preimage of $j$ for the unique monotone bijection $\langle n-|A|\rangle \stackrel{\approx}{\longrightarrow}\langle n\rangle \backslash A$.

An $n$-cube of $\Gamma$-groups $\mathfrak{F}$ determines a normal $(n+1)$-ad of $\Gamma$-groups $\left(\mathfrak{F}(\emptyset) ; R_{1}\right.$, $\left.\cdots, R_{n}\right)$, where $R_{i}=\operatorname{Ker} \mathfrak{F}\left(\rho_{\{i\}}^{\emptyset}\right), i \in\langle n\rangle$. This $(n+1)$-ad will be called the normal $(n+1)$-ad of $\Gamma$-groups induced by $\mathfrak{F}$.

Given an $n$-cube of $\Gamma$-groups $\mathfrak{F}$, it is easy to see that there exists a natural $\Gamma$-homomorphism $\mathfrak{F}(A) \stackrel{\alpha_{A}}{\longrightarrow} \lim _{B \supset A} \mathfrak{F}(B)$ for any $A \subseteq\langle n\rangle, A \neq\langle n\rangle$.

Definition 7. An $n$-cube of $\Gamma$-groups $\mathfrak{F}$ will be called an $n$-presentation of a $\Gamma$ group $G$ if $\mathfrak{F}(\langle n\rangle)=G$. An $n$-presentation $\mathfrak{F}$ of $G$ is called projective if $\mathfrak{F}(A)$ is a projective $\Gamma$-group for all $A \neq\langle n\rangle$, and it is called exact if every $\alpha_{A}(A \subseteq\langle n\rangle$, $A \neq\langle n\rangle)$ which has a section map preserving the $\Gamma$-actions is surjective.

The following lemma is straightforward.

Lemma 8. An augmented simplicial $\Gamma$-group $\left(G_{*}, d_{0}^{0}, G\right)$ with $\pi_{0}\left(G_{*}\right) \cong G$ is a projective simplicial resolution of $G$ if and only if the $n$-cube of $\Gamma$-groups $\mathfrak{F}^{(n)}\left(G_{*}, d_{0}^{0}, G\right)$ is a projective exact $n$-presentation of $G$ for all $n \geq 1$.

Given a homomorphism of $\Gamma$-groups $\alpha: H \rightarrow G$, the Čech augmented complex for $\alpha$ is the augmented simplicial $\Gamma$-group $\left(\check{C}(\alpha)_{*}, \alpha, G\right)$ defined by

$$
\begin{aligned}
& \check{C}(\alpha)_{n}=\underbrace{H \times_{G} \cdots \times_{G} H}_{(n+1) \text { times }}=\left\{\left(h_{0}, \ldots, h_{n}\right) \in H^{n+1} \mid \alpha\left(h_{0}\right)=\cdots=\alpha\left(h_{n}\right)\right\}, \\
& d_{i}^{n}\left(h_{0}, \ldots, h_{n}\right)=\left(h_{0}, \ldots, \hat{h}_{i}, \ldots, h_{n}\right), \\
& s_{i}^{n}\left(h_{0}, \ldots, h_{n}\right)=\left(h_{0}, \ldots, h_{i}, h_{i}, h_{i+1}, \ldots, h_{n}\right),
\end{aligned}
$$

for $0 \leq i \leq n$.

Now let $\mathfrak{F}$ be an $n$-presentation of the $\Gamma$-group $G$. Applying $\check{C}$ above, in the $n$-independent directions, this construction leads naturally to an augmented $n$ simplicial $\Gamma$-group. Taking the diagonal of this augmented $n$-simplicial $\Gamma$-group gives the augmented simplicial $\Gamma$-group $\left(\check{C}^{(n)}(\mathfrak{F})_{*}, \alpha, G\right)$ called an augmented $n$-fold Čech complex for $\mathfrak{F}$, where $\alpha=\mathfrak{F}\left(\rho_{\langle n\rangle}^{\emptyset}\right): \mathfrak{F}(\emptyset) \rightarrow G$. In case $\mathfrak{F}$ is a projective exact $n$-presentation of $G$, then $\left(\check{C}^{(n)}(\mathfrak{F})_{*}, \alpha, G\right)$ will be called an $n$-fold $\check{C}$ ech resolution of $G$.

Definition 9. Let $T: \mathcal{G}_{\Gamma} \rightarrow \mathcal{G}$ be a covariant functor. Define $k$-th $n$-fold Čech

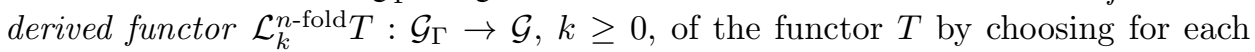
$\Gamma$-group $G$, a projective exact $n$-presentation $\mathfrak{F}$ and setting

$$
\mathcal{L}_{k}^{n \text {-fold }} T(G)=\pi_{k}\left(T \check{C}^{(n)}(\mathfrak{F})_{*}\right),
$$

where $\left(\check{C}^{(n)}(\mathfrak{F})_{*}, \alpha, G\right)$ is the $n$-fold Čech resolution of the $\Gamma$-group $G$ for the projective exact $n$-presentation $\mathfrak{F}$ of $G$. 
Note that by [12] (see also [7, Theorem 16]) the $n$-fold Čech derived functors are well defined. Moreover, if $F$ is a projective $\Gamma$-group, then

$$
\mathcal{L}_{k}^{n \text {-fold }} T(F)=1, \quad n \geq 1 .
$$

Lemma 10. Let $\mathfrak{F}$ be an n-presentation of a $\Gamma$-group $G$. There is an isomorphism of simplicial $\Gamma$-groups

$$
E_{\Gamma}^{n}(\mathcal{M})_{*} \cong \check{C}^{(n)}(\mathfrak{F})_{*},
$$

where $\mathcal{M}$ is the inclusion crossed $n$-cube of $\Gamma$-groups given by the normal $(n+1)$-ad of $\Gamma$-groups $\left(\mathfrak{F}(\emptyset) ; R_{1}, \ldots, R_{n}\right), R_{i}=\operatorname{Ker} \mathfrak{F}\left(\rho_{\{i\}}^{\emptyset}\right), i \in\langle n\rangle$.

Proof. For $n=1$ the required isomorphism

$$
\lambda_{*}: E_{\Gamma}^{1}\left(R_{1} \hookrightarrow \mathfrak{F}(\emptyset)\right)_{*} \stackrel{\approx}{\longrightarrow} \check{C}(\mathfrak{F}(\emptyset) \rightarrow G)_{*}
$$

is given by $\lambda_{0}=i d_{\mathfrak{F}(\emptyset)}$ and $\lambda_{k}\left(r_{1}, \cdots, r_{k}, f\right)=\left(r_{1} \ldots r_{k} f, r_{2} \ldots r_{k} f, \cdots, r_{k} f, f\right)$ for all $k \geq 1$ and $\left(r_{1}, \cdots, r_{k}, f\right) \in R_{1} \rtimes \cdots \rtimes R_{1} \rtimes \mathfrak{F}(\emptyset)$.

Then by repeated applications of this isomorphism, we get an isomorphism of $n$-simplicial $\Gamma$-groups. Applying the diagonal gives the result for any $n$.

Now we give an explicit computation of the $n$-th $n$-fold Čech derived functor of the $\Gamma$-abelianization functor $\mathfrak{A b}_{\Gamma}: \mathcal{G}_{\Gamma} \rightarrow \mathcal{A} b$, implying a description of the equivariant integral homology of groups from the point of view of Hopf type formulas.

Theorem 11. Let $G$ be a $\Gamma$-group and $\mathfrak{F}$ its projective exact $n$-presentation. Then there is an isomorphism

$$
\mathcal{L}_{n}^{n-f o l d} \mathfrak{A b}_{\Gamma}(G) \cong \frac{\bigcap_{i \in\langle n\rangle} R_{i} \cap[F, F]_{\Gamma}}{\left(\prod_{A \subseteq\langle n\rangle}\left[\bigcap_{i \in A} R_{i}, \bigcap_{i \notin A} R_{i}\right]\right)\left[\bigcap_{i \in\langle n\rangle} R_{i}\right]_{\Gamma}}, \quad n \geq 1,
$$

where $F=\mathfrak{F}(\emptyset)$ and $R_{i}=\operatorname{Ker}(\mathfrak{F}(\emptyset) \rightarrow \mathfrak{F}(\{i\}))$ for $i \in\langle n\rangle$.

Proof. Using Lemma 10, $\mathcal{L}_{n}^{n \text {-fold }} \mathfrak{A b}_{\Gamma}(G) \cong \pi_{n}\left(\mathfrak{A b}_{\Gamma} E_{\Gamma}^{n}(\mathcal{M})_{*}\right)$, where $\mathcal{M}$ is the inclusion crossed $n$-cube of $\Gamma$-groups induced by the normal $(n+1)$-ad of $\Gamma$-groups $\left(F ; R_{1}, \cdots, R_{n}\right)$. Hence Proposition 5 implies an isomorphism $\mathcal{L}_{n}^{n \text {-fold }} \mathfrak{A b}_{\Gamma}(G) \cong$ $\pi_{n}\left(E_{\Gamma}^{n} \mathfrak{A} \mathfrak{b}_{\Gamma}^{n}(\mathcal{M})_{*}\right)$. Then, by [7, Proposition 14] (see also [14, Proposition 3.4]), there is an isomorphism

$$
\mathcal{L}_{n}^{n \text {-fold }} \mathfrak{A b}_{\Gamma}(G) \cong \bigcap_{l \in\langle n\rangle} \operatorname{Ker}\left(\mathfrak{A b}_{\Gamma}^{n}(\mathcal{M})_{\langle n\rangle} \stackrel{\widetilde{\mu_{l}}}{\longrightarrow} \mathfrak{A b}_{\Gamma}^{n}(\mathcal{M})_{\langle n\rangle \backslash\{l\}}\right),
$$

where, by definition of the functor $\mathfrak{A b}_{\Gamma}{ }_{\Gamma}$, we have

$$
\mathfrak{A b}_{\Gamma}^{n}(\mathcal{M})_{A}=\frac{\bigcap_{i \in A} R_{i}}{\left(\prod_{B \subseteq A}\left[\bigcap_{i \in B} R_{i}, \bigcap_{i \notin B} R_{i}\right]\right)\left[\bigcap_{i \in A} R_{i}\right]_{\Gamma}}, \quad A \subseteq\langle n\rangle .
$$

Now we set up the inductive hypothesis. Let $n=1$. Then

$$
\mathcal{L}_{1}^{1 \text {-fold }} \mathfrak{A b}_{\Gamma}(G) \cong \operatorname{Ker}\left(\frac{R_{1}}{\left[F, R^{1}\right]_{\Gamma}} \longrightarrow \frac{F}{[F, F]_{\Gamma}}\right)=\frac{R_{1} \cap[F, F]_{\Gamma}}{\left[F, R_{1}\right]_{\Gamma}} .
$$

Proceeding by induction, suppose the result is true for $n-1$; we will prove it for $n$. 
Let $l \in\langle n\rangle$ and denote by $\mathfrak{F}^{\overline{\{l\}}}$ the restriction of the functor $\mathfrak{F}: \mathcal{C}_{n} \rightarrow \mathcal{G}_{\Gamma}$ to the subcategory of $\mathcal{C}_{n}$ consisting of all subsets $A \subseteq\langle n\rangle$ with $l \notin A$. It is easy to check that $\mathfrak{F}^{\{l\}}$ is a projective exact $(n-1)$-presentation of the projective $\Gamma$-group $\mathfrak{F}(\langle n\rangle \backslash\{l\})$. By (2) we have $\mathcal{L}_{n-1}^{(n-1) \text {-fold }} \mathfrak{A b}_{\Gamma}(\mathfrak{F}(\langle n\rangle \backslash\{l\}))=1$. Then our inductive hypothesis implies that

$$
\bigcap_{i \in\langle n\rangle \backslash\{l\}} R_{i} \cap[F, F]_{\Gamma}=\left(\prod_{A \subseteq\langle n\rangle \backslash\{l\} \in A}\left[\bigcap_{i \notin A} R_{i}, \bigcap_{i \notin A} R_{i}\right]\right)\left[\bigcap_{i \in\langle n\rangle \backslash\{l\}} R_{i}\right]_{\Gamma} .
$$

Then from the equalities (3) and (4) we deduce the required isomorphism.

Finally we provide the main theorem, which expresses the equivariant integral homology of groups as Hopf type formulas generalizing the Brown-Ellis result for trivial actions [2] and extending to higher dimensions the Hopf formula for the second equivariant group homology [11].

Theorem 12. Let $G$ be $a \Gamma$-group and $\mathfrak{F}$ its projective exact $n$-presentation. Then there is an isomorphism

$$
H_{n+1}^{\Gamma}(G) \cong \frac{\bigcap_{i \in\langle n\rangle} R_{i} \cap[F, F]_{\Gamma}}{\left(\prod_{A \subseteq\langle n\rangle}\left[\bigcap_{i \in A} R_{i}, \bigcap_{i \notin A} R_{i}\right]\right)\left[\bigcap_{i \in\langle n\rangle} R_{i}\right]_{\Gamma}}, \quad n \geq 1,
$$

where $F=\mathfrak{F}(\emptyset)$ and $R_{i}=\operatorname{Ker}(\mathfrak{F}(\emptyset) \rightarrow \mathfrak{F}(\{i\}))$ for $i \in\langle n\rangle$.

Proof. Let $\left(F_{*}, d_{0}^{0}, G\right)$ be a projective simplicial resolution of the $\Gamma$-group $G$ and consider the short exact sequence of augmented simplicial $\Gamma$-groups

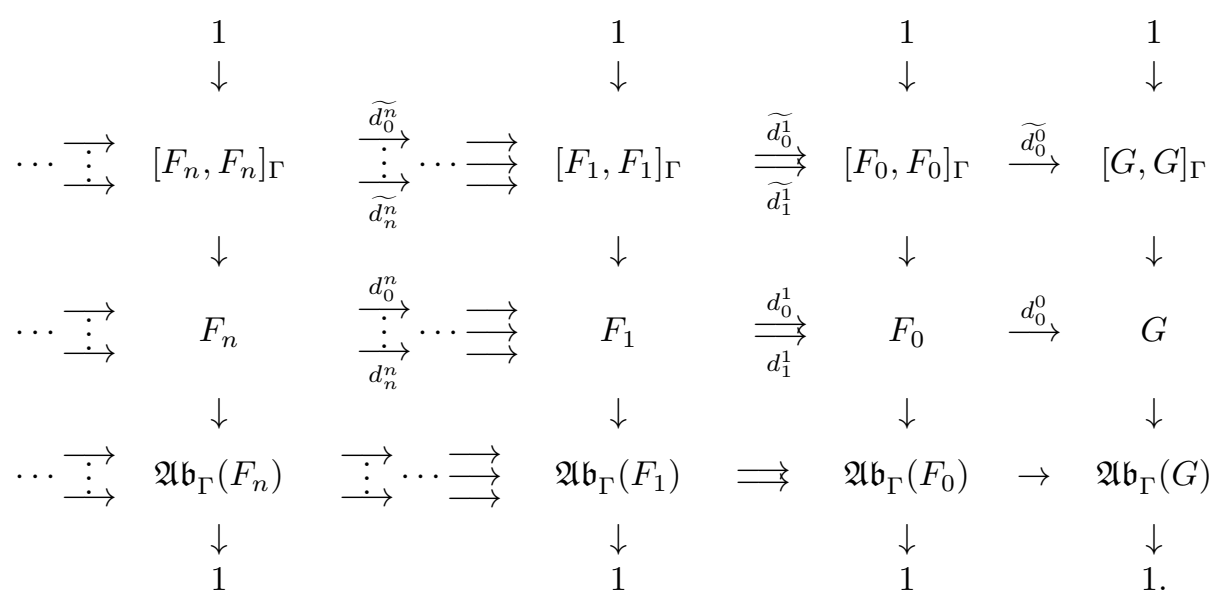

By the induced long exact homotopy sequence, there is an isomorphism

$$
\pi_{n} \mathfrak{A} \mathfrak{b}_{\Gamma}\left(F_{*}\right) \cong \frac{\bigcap_{i \in\langle n\rangle} \operatorname{Ker} \widetilde{d_{i-1}^{n-1}}}{\widetilde{d_{n}^{n}}\left(\bigcap_{i \in\langle n\rangle} \operatorname{Ker} \widetilde{d_{i-1}^{n}}\right)}, \quad n \geq 1 .
$$

Since $\widetilde{d_{i-1}^{n}}$ is the restriction of $d_{i-1}^{n}$ to $\left[F_{n}, F_{n}\right]_{\Gamma}$, Ker $\widetilde{d_{i-1}^{n}}=\operatorname{Ker} d_{i-1}^{n} \cap\left[F_{n}, F_{n}\right]_{\Gamma}$. Hence $\bigcap_{i \in\langle n\rangle} \operatorname{Ker} \widetilde{d_{i-1}^{n}}=\left(\bigcap_{i \in\langle n\rangle} \operatorname{Ker} d_{i-1}^{n}\right) \cap\left[F_{n}, F_{n}\right]_{\Gamma}$ and similarly $\bigcap_{i \in\langle n\rangle} \operatorname{Ker} \widetilde{d_{i-1}^{n-1}}$ $=\left(\bigcap_{i \in\langle n\rangle} \operatorname{Ker} d_{i-1}^{n-1}\right) \cap\left[F_{n-1}, F_{n-1}\right]_{\Gamma}$. 
Since the shift of the simplicial object $F_{*}$ is the contractible augmented simplicial object $\left(\operatorname{Dec}\left(F_{*}\right), d_{0}^{1}, F_{0}\right)$ (see [8]), by Lemma 8, the $n$-cube of $\Gamma$-groups $\mathfrak{F}^{(n)}\left(\operatorname{Dec}\left(F_{*}\right), d_{0}^{1}, F_{0}\right)$ is a projective exact $n$-presentation of the $\Gamma$-group $F_{0}$. Hence, by Theorem [1] and the equality (2) we have

$$
\mathcal{L}_{n}^{n \text {-fold }} \mathfrak{A b}_{\Gamma}\left(F_{0}\right) \cong \frac{\bigcap_{i \in\langle n\rangle} \operatorname{Ker} d_{i-1}^{n} \cap\left[F_{n}, F_{n}\right]_{\Gamma}}{\left(\prod_{A \subseteq\langle n\rangle}\left[\bigcap_{i \in A} \operatorname{Ker} d_{i-1}^{n}, \bigcap_{i \notin A} \operatorname{Ker} d_{i-1}^{n}\right]\right)\left[\bigcap_{i \in\langle n\rangle} \operatorname{Ker} d_{i-1}^{n}\right]_{\Gamma}}=1,
$$

$$
n \geq 1,
$$

implying that

(6)

$$
\begin{array}{r}
\bigcap_{i \in\langle n\rangle} \operatorname{Ker} d_{i-1}^{n} \cap\left[F_{n}, F_{n}\right]_{\Gamma}=\left(\prod_{A \subseteq\langle n\rangle}\left[\bigcap_{i \in A} \operatorname{Ker} d_{i-1}^{n}, \bigcap_{i \notin A} \operatorname{Ker} d_{i-1}^{n}\right]\right)\left[\bigcap_{i \in\langle n\rangle} \operatorname{Ker} d_{i-1}^{n}\right]_{\Gamma}, \\
n \geq 1 .
\end{array}
$$

Since $\left(F_{*}, d_{0}^{0}, G\right)$ is a projective simplicial resolution of $G, d_{n}^{n}\left(\bigcap_{i \in\langle n\rangle} \operatorname{Ker} d_{i-1}^{n}\right)=$ $\bigcap_{i \in\langle n\rangle} \operatorname{Ker} d_{i-1}^{n-1}, n \geq 1$. It follows that $d_{n}^{n}\left(\left[\bigcap_{i \in\langle n\rangle} \operatorname{Ker} d_{i-1}^{n}\right]_{\Gamma}\right)=\left[\bigcap_{i \in\langle n\rangle} \operatorname{Ker} d_{i-1}^{n-1}\right]_{\Gamma}$. Using this fact and the equality (6) it is easy to see that

$$
\begin{aligned}
\widetilde{d_{n}^{n}}\left(\bigcap_{i \in\langle n\rangle} \operatorname{Ker} \widetilde{d_{i}^{n}}\right) & =d_{n}^{n}\left(\left(\prod_{A \subseteq\langle n\rangle}\left[\bigcap_{i \in A} \operatorname{Ker} d_{i-1}^{n}, \bigcap_{i \notin A} \operatorname{Ker} d_{i-1}^{n}\right]\right)\left[\bigcap_{i \in\langle n\rangle} \operatorname{Ker} d_{i-1}^{n}\right]_{\Gamma}\right) \\
& =\left(\prod_{A \subseteq\langle n\rangle}\left[\bigcap_{i \in A} \operatorname{Ker} d_{i-1}^{n-1}, \bigcap_{i \notin A} \operatorname{Ker} d_{i-1}^{n-1}\right]\right)\left[\bigcap_{i \in\langle n\rangle} \operatorname{Ker} d_{i-1}^{n-1}\right]_{\Gamma} .
\end{aligned}
$$
have

Then, taking into account the equality (11) and Remark 2 , by the equality (5) we

$$
H_{n+1}^{\Gamma}(G) \cong \frac{\left(\bigcap_{i=0}^{n-1} \operatorname{Ker} d_{i}^{n-1}\right) \cap\left[F_{n-1}, F_{n-1}\right]_{\Gamma}}{\left(\prod_{A \subseteq\langle n\rangle}\left[\bigcap_{i \in A} \operatorname{Ker} d_{i-1}^{n-1}, \bigcap_{i \notin A} \operatorname{Ker} d_{i-1}^{n-1}\right]\right)\left[\bigcap_{i \in\langle n\rangle} \operatorname{Ker} d_{i-1}^{n-1}\right]_{\Gamma}} .
$$

Using again Lemma 8 and Theorem 11 completes the proof.

\section{REFERENCES}

[1] M. Barr and J. Beck, Homology and standard constructions, Lecture Notes in Mathematics, Vol. 80, Springer-Verlag, Berlin/New York (1969), 245-335. MR0258917 (41:3562)

[2] R. Brown and G. J. Ellis, Hopf formulae for the higher homology of a group, Bull. London Math. Soc. 20 (1988), 124-128. MR.924238 (89g:20082)

[3] G. Carlsson, Equivariant stable homotopy theory and related areas, in: G. Carlsson (ed.), Proc. Workshop at Stanford University, 2000, Homology Homotopy Appl. 3 (2) (2001), viixv. MR 1856027

[4] J. M. Casas, N. Inassaridze, E. Khmaladze and M. Ladra, Homology of $(n+1)$-types and Hopf type formulas, J. Pure Appl. Algebra 200 (2005), 267-280. MR2147270 (2006b:18014)

[5] A.M. Cegarra, J.M. Garcia-Calcines, and J.A. Ortega, Cohomology of groups with operators, Homology Homotopy Appl. 4 (1) (2002), 1-23. MR.1894065 (2003c:20064)

[6] A.M. Cegarra and H. Inassaridze, Homology of groups with operators, Int. Math. J. 5 (1) (2004), 29-48. MR 2031933

[7] G. Donadze, N. Inassaridze and T. Porter, $n$-Fold Čech derived functors and generalised Hopf type formulas, K-theory 35 (2003), 341-373. MR2240237 (2007d:18022) 
[8] J. Duskin, Simplicial methods and the interpretation of "triple" cohomology, Mem. Amer. Math. Soc. 3 (1975), no. 163. MR0393196 (52:14006)

[9] G.J. Ellis and R. Steiner, Higher-dimensional crossed modules and the homotopy groups of $(n+1)-a d s$, J. Pure Appl. Algebra 46 (1987), 117-136. MR897011 (88j:55010)

[10] Z. Fiedorowicz, H. Hauschild and J.P. May, Equivariant K-theory, Lecture Notes in Math., 967, Springer, Berlin, 1982, 23-80. MR689388 (84h:57023)

[11] H. Inassaridze, Equivariant homology and cohomology of groups, Topology and its Application, 153 (2005), 66-89. MR2172035 (2006e:18020)

[12] N. Inassaridze, $N$-fold Čech derived functors of group valued functors, Bull. Georgian Acad. Sci. 168 (2) (2003).

[13] A. Kuku, Equivariant K-theory and the cohomology of profinite groups, Algebraic K-Theory, Number Theory, Geometry and Analysis, in: Lecture Notes in Math., 342, Springer, Berlin, 1984, 235-244. MR750684 (86h:18005)

[14] J.-L. Loday, Spaces with finitely many nontrivial homotopy groups, J. Pure Appl. Algebra 24 (1982), 179-202. MR651845 (83i:55009)

[15] N.C. Philips, Equivariant K-theory and freeness of group actions on $C^{*}$-algebras, Lecture Notes in Math., 1274, Springer, Berlin, 1987. MR.911880 (89k:46086)

Department of Algebra, A. Razmadze Mathematical Institute, M. Alexidze St. 1, 0193 Tbilisi, Georgia - And - Tbilisi Centre for Mathematical Sciences, Tbilisi, Georgia

E-mail address: hvedri@rmi.acnet.ge

Department of Algebra, A. Razmadze Mathematical Institute, M. Alexidze St. 1, 0193 Tbilisi, Georgia - and - Tbilisi Centre for Mathematical Sciences, Tbilisi, Georgia

E-mail address: khmal@rmi.acnet.ge 